\title{
Adaptive Interference Mitigation with User Grouping for Fast Transmission in Cellular Networks
}

\author{
Hadhrami Ab. Ghani*1, Azlan Abd Aziz', Azizul Azizan', Salwani Mohd Daud ${ }^{4}$ \\ ${ }^{1,2}$ Faculty of Engineering and Technology, Multimedia University, Malaysia \\ 3,4 Advanced Informatics School, Universiti Teknologi Malaysia \\ ${ }^{3}$ Wireless Communication Centre, Universiti Teknologi Malaysia
}

\begin{tabular}{l} 
Article Info \\
\hline Article history: \\
Received Nov 23, 2017 \\
Revised Jan 28, 2018 \\
Accepted Feb 22, 2018 \\
\hline Keywords: \\
Energy efficiency \\
Error rate reduction \\
Fast transmission \\
Interference mitigation \\
User grouping
\end{tabular}

\begin{abstract}
Designing uplink systems which group users with adaptive interference mitigation techniques is the objective of this research. Reduction in error rates and improvement in the energy efficiency is expected with this approach in addition to spectral efficiency. This paper reports a study on interference mitigation and transmission designs for groups of users in the uplinks. New formulations for the interference mitigation are produced based on the minimum mean square error and successive interference cancellation approach. By reducing the interference, the energy efficiency can be maintained and improved although the number of users per group increases. The measured error rates of this approach with user grouping achieve gains between 1 to $3 \mathrm{~dB}$ against that of the existing approach. With reduced complexity, the proposed scheme should be viable for practical deployment.
\end{abstract}

Copyright $@ 2018$ Institute of Advanced Engineering and Science. All rights reserved.

\section{Corresponding Author:}

Hadhrami Ab. Ghani,

Faculty of Engineering and Technology,

Multimedia University,

Jalan Ayer Keroh Lama, 75450 Bukit Beruang, Melaka, Malaysia.

Email: hadhrami.abdghani@mmu.edu.my

\section{INTRODUCTION}

The speedy increase in the achievable data rates of the latest cellular network technologies has resulted in significant advantages to all Internet users, including both home and business users. Without this outstanding data rates offered by the cellular network providers, the various smart phones or devices manufactured for billions of people on the planet will be in vain. However, the growing trend in the achievable data rates has further sparked the increasing demand for higher speed cellular data services which will be served at the price of better or higher energy and spectral efficiency. The majorlimitation of energy and spectral efficiency is the interference between symbols, carriers and users [1-3]. Although spectrally efficient Orthogonal Frequency Division Multiplexing (OFDM) systems are applied in current cellular networks such as long term evolution (LTE)-Advanced networks [4-5], inter-carrier interference may increase due to several degrading factors including Doppler effects. Hence, to efficiently utilize the carrier bandwidth [6-7], interference mitigation schemes should be implemented at the receiving end.

With interference cancellation, the shared carrier bandwidth amongst the single-antenna users in a virtual multiple input multiple output (MIMO) scenario, as proposed in (e.g.[8,9]) further increases the spectral efficiency. Interference cancellation schemes such as successive interference cancellation (SIC) and parallel interference cancellation (PIC) schemes are traditionally designed with coding and/or equalization techniques such as minimum mean square error (MMSE) to remove interference $[1,2]$. Multiple-feedback (MF) SIC is also proposed to mitigate interference using multiple feedbacks at the receiver. Although the conventional MMSE-SIC and power allocation improve the energy efficiency [10-12], the incurred computational complexity tends to increase when the number of users increases. The MF-SIC scheme offers 
lower computational complexity as compared to the MMSE-SIC scheme. However the increasing number of multiple feedbacks received during the detection process will also increase the computational complexity. A multicast communication approach [13] has been proposed to reduce the complexity but no specific solutions on other communication modes are given.

In this paper, a new simplified MMSE-SIC receiver scheme for high speed transmission systems like LTE-Avanced systems is proposed to detect groups of users in multi-user MIMO OFDM systems which are widely used in the current and future cellular networks.

\section{RESEARCH METHOD}

Consider a high-speed MIMO OFDM model with $N_{\text {user }}$ users, each with a single antenna and communicates over the uplinks with the base station having $N_{r}$ antennas and $M$ frequency sub-carriers. The block diagram for the corresponding transmitter and receiver models is shown in Figure 1. The focus of this paper is at the receiving part, which is indicated in the diagram using italic fonts, which are the adaptive SIC with grouping. Before explaining about this proposed method, the details of the cellular network set up are given in this section. The network is isometric, which means that all users are equally distant to the base station and the channel state information (CSI) is not available at the receiving side. The OFDM symbols are generated using $N$-IDFT, as seen in Figure 1, where $N=2048$ has been widely used for LTE systems. With $N_{u}$ users per group, the number of groups is $N_{G}=N_{u s e r} / N_{u}$ where the $p$-th user of the $j$-th group is represented as $u(j, p)$, with $j=1,2, \cdots, N_{G}$ and $p=1,2, \ldots, N_{u}$. The received vector $\mathbf{r}_{j} \in \mathbb{C}^{N_{r} N \times 1}$ for group $j$ is

$$
\mathbf{r}_{j}=\mathbf{H}_{j}\left[\begin{array}{c}
\boldsymbol{\Psi}_{j} \mathbf{a}_{u(j, 1)} \\
\vdots \\
\boldsymbol{\Psi}_{j} \mathbf{a}_{u\left(j, N_{u}\right)}
\end{array}\right]+\left[\begin{array}{c}
\mathbf{n}_{1} \\
\vdots \\
\mathbf{n}_{N_{r}}
\end{array}\right]
$$

with $\boldsymbol{\Psi}_{j} \in \mathbb{C}^{N \times M}$ as the matrix for mapping the frequency carriers to the users in group $j$ for $\forall j=$ $1,2, \cdots, N_{G}$. The noise vector is represented as $\mathbf{n}_{j} \in \mathbb{C}^{N \times 1}$. Matrix $\mathbf{H}_{j} \in \mathbb{C}^{N_{r} N \times N_{u} N}$ represents the received channel gains of group $j$ where $\mathbf{H}_{j} \triangleq\left[\mathbf{H}_{j, 1} \cdots \mathbf{H}_{j, N_{u}}\right]$. Matrix $\mathbf{H}_{j, p} \in \mathbb{C}^{N_{r} N \times N}$ is then defined as the received channel matrix of the $p$-th user from the $j$-th group, which is not known at the receiving end. After the modulation and coding process, the data vector $\mathbf{a}_{u(j, p)} \in \mathbb{C}^{M \times 1}$ from user $p$ of group $j$ is mapped to the frequency domain via the Fourier Transform operation. In order to remove the inter symbol interference, the cyclic prefix operation is also performed before transmission and after the data vector is received.

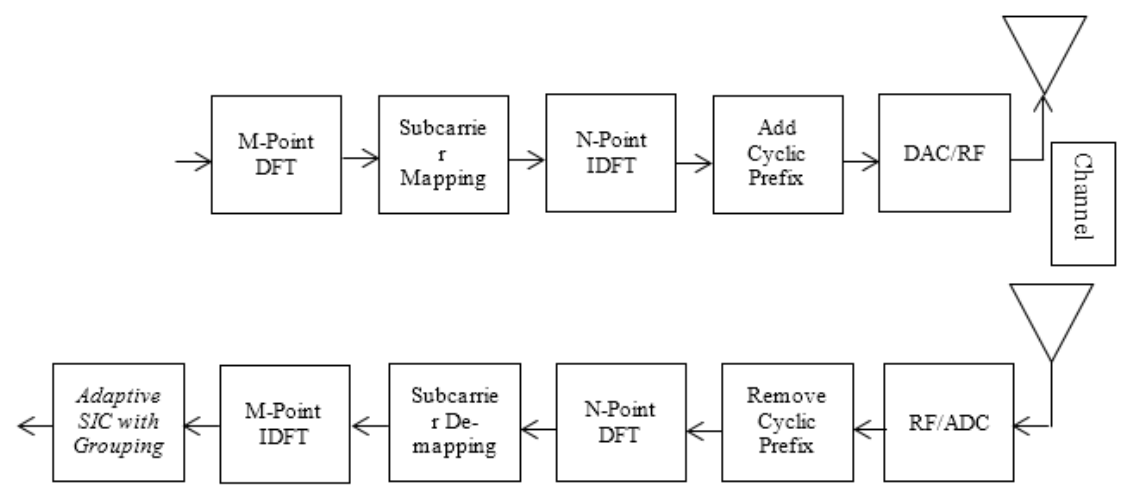

Figure 1: The Transmitter and Receiver Model.

When the transmitted signal of each user has an equal power spectral density, $\sigma_{a, u(j, p)}^{2}$, corrupted by additive white Gaussian noise with a spectral density, $\sigma_{n, u(j, p)}^{2}$, the input signal-to-noise ratio (SNR) can be represented as $\rho_{u(j, p)}=\sigma_{a, u(j, p)}^{2} / \sigma_{n, u(j, p)}^{2}$. By using the MMSE approach, a detection matrix [9], $\mathbf{F}_{j, \iota_{p}} \in$ $\mathbb{C}^{M \times N_{r} N}$, for recovering the received signal can be derived to produce the following expression

$$
\mathbf{F}_{j, p}=\boldsymbol{\Psi}_{j}^{H} \mathbf{H}_{j, p}^{H} \mathbf{V}_{j, p}\left(\mathbf{H}_{j} \mathbf{H}_{j}^{H}+\rho_{u(j, p)}^{-1} \mathbf{I}_{N_{r} N}\right)^{-1},
$$


where $\mathbf{V}_{j, p}=\frac{1}{\sigma_{a, u(j, p)}^{2}} \mathbf{I}_{N_{r} N}$

The interference mitigation technique proposed in this paper is based on the successive interference cancellation method. By updating the effective amount of interference experienced by the current user received data to be detected, Equation (2) which represents the inverse covariance matrix must be recalculated whenever a received data vector is successfully detected in order to exclude the amount of interference contributed by the received data which have been successfully recovered. This updated inverse matrix, $\mathbf{D}_{j, \iota_{p}} \in \mathbb{R}^{N_{r} N \times N_{r} N}$, can be expressed as follows

$$
\mathbf{D}_{j, \iota_{p}}=\left(\mathbf{H}_{j, \iota_{1}} \mathbf{H}_{j, \iota_{1}}^{H}+\cdots+\mathbf{H}_{j, \iota_{p}} \mathbf{H}_{j, \iota_{p}}^{H}+\rho_{u\left(j, \iota_{p}\right)}^{-1} \mathbf{I}_{N_{r} N}\right)^{-1},
$$

where $\iota_{p}$ denotes the $p$-th user of the $j$-th group. The received channel matrices (eg. $\mathbf{H}_{j, \iota_{p}} \mathbf{H}_{j, \iota_{p}}^{H}$ ) refer to those users $\iota_{1}, \iota_{2}$ until $\iota_{p}$, which will be detected, excluding the users which have been successfully detected. Hence, a new matrix which is used to perform interference mitigation for detecting the users can be written as

$$
\mathbf{F}_{j, l_{p}}=\boldsymbol{\Psi}_{j}^{H} \mathbf{H}_{j, \iota_{p}}^{H} \mathbf{V}_{j, \iota_{p}} \mathbf{D}_{j, \iota_{p}},
$$

The matrix in Equation (4) can be used to detect the data vector received from user $\iota_{p}$, which is expressed as $\hat{\mathbf{a}}_{u\left(j, \iota_{p}\right)}=\mathbf{F}_{j, \iota_{p}} \hat{\mathbf{r}}_{j, \iota_{p}}$, where vector $\hat{\mathbf{r}}_{j, \iota_{p}}$ contains interference only from the later users which have not been detected, and is calculated as shown below [14]

$$
\hat{\mathbf{r}}_{j, \iota_{p}-1}=\hat{\mathbf{r}}_{j, \iota_{p}}-\mathbf{H}_{j, \iota_{p}} \boldsymbol{\Psi}_{j} \hat{\mathbf{a}}_{u\left(j, \iota_{p}\right)},
$$

In this paper, a new formulation is produced based on the successive interference cancellation approach to produce a computationally lower transmission model. The main computations are involved in determining matrix $\mathbf{D}_{j, \iota_{p}}$ of Equation (3), which is the inverse covariance matrix. For notational convenience, the entry of this matrix at row $x$ and column $y$ is expressed as $D_{j, \iota_{p}}=\left[\mathbf{D}_{j, \iota_{p}}\right]_{x, y}$, which is further detailed as follows [14]

$$
D_{j, \iota_{p}}=\left\{\begin{array}{cc}
{\left[\mathbf{C}_{j, \iota_{p}, n}^{-1}\right]_{r+1, s+1},} & \text { if } \frac{x-r N}{y-s N}=1 \\
0, & \text { elsewhere }
\end{array}\right.
$$

with $n=x-r N=y-s N$ for $n=1, \cdots, N$ and $r, s=0,1, \cdots, N_{r}-1$. In the received channel matrix, the entry is represented as, $H_{j, x, y}=\left[\mathbf{H}_{j}\right]_{x, y}$. Hence the covariance matrix is rewritten as below [14]

$$
\mathbf{C}_{j, \iota_{p}, n}=\left[\begin{array}{ccc}
S_{j, n, n}^{\left(\iota_{p}\right)} & \cdots & \zeta_{j, n, n+N_{r} N}^{\left(\iota_{p}\right)} \\
\vdots & \ddots & \vdots \\
\zeta_{j, n+N_{r} N, n}^{\left(\iota_{p}\right)} & \cdots & \zeta_{j, n+N_{r} N, n+N_{r} N}^{\left(\iota_{p}\right)}
\end{array}\right]
$$

where

$$
\begin{aligned}
S_{j, x, y}^{\left(\iota_{p}\right)} & =\sum_{p=1}^{\iota_{p}} H_{j, x, l} H_{j, x, l}^{*}+\rho^{-1} v(x, y), \\
& =\varsigma_{j, x, y}^{\left(\iota_{p}-1\right)}+H_{j, x, n+\left(\iota_{p}-1\right) N} H_{j, y, n+\left(\iota_{p}-1\right) N .}^{*}
\end{aligned}
$$

with $v(x, y)$ value is unity when $x=y$ and zero otherwise. The successive interference cancellation operation is started from the $N_{u}$-th user, which is interfered by transmitted data from all users. Hence the detection of this user, which is indicated as $\iota_{p}=N_{u}$, is similar to the MMSE detection process, which is also simplified and presented in this paper. To complete the interference mitigation process after detecting the $N_{u^{-}}$ th user, the interference caused by this newly detected user is removed and the next user i.e. user $N_{u}-1$ is 
then detected. The same process continues for all users. Hence, the entries of the interference mitigation matrix $\mathbf{F}_{j, \iota_{p}}$ for recovering the received data is written as

$$
F_{j, m, y+r N}=\frac{1}{\sigma_{a}^{2}} \psi_{j, m, y}^{*} \sum_{s=0}^{N_{r}-1} H_{j, y+r N, y+s N}^{*} D_{j, y+r N, y+s N}
$$

where $F_{j, m, y+r N}=\left[\mathbf{F}_{j, \iota_{p}}\right]_{n, y+r N}, \psi_{j, m, y}=\left[\boldsymbol{\Psi}_{j}\right]_{m, y}$ for $r=0,1, \ldots, N_{r}$ and $m=v_{\text {offset }}+1, \ldots, v_{\text {offset }}+M$. In the next section, a brief analysis on the spectral and energy efficiency is carried out to measure performance of the users in the proposed system.

\subsection{Energy and Spectral Efficiency}

The energy efficiency is determined as follows

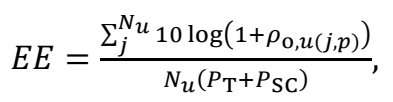

where $\rho_{\mathrm{o}, u(j, p)}$ is the unbiased signal-to-interference and noise ratio (SINR) [8] given by $\rho_{\mathrm{o}, u(j, p)}=$ $\frac{\sigma_{a, u(j, p)} \mathbf{h}^{\boldsymbol{H}}{ }_{j, z} \mathbf{D}_{j, p} \mathbf{h}_{j, z}}{1-\sigma_{a, u(j, p)} \mathbf{h}^{\boldsymbol{H}}{ }_{j, z} \mathbf{D}_{j, p} \mathbf{h}_{j, z}}$, where $\mathbf{h}_{j, z}$ is the $z$-th column of $\mathbf{H}_{j}$, index $z$ is given as $z=v_{o}+(p-1) N$ and $v_{o}$ is the frequency carrier offset, $P_{\mathrm{T}}$ is the transmit power and $P_{\mathrm{SC}}$ is the load-independent circuit power. Another important measurement parameter is the bandwidth efficiency, written as

$$
S E=\frac{\sum_{j}^{N u} 10 \log \left(1+\rho_{0, u(j, p)}\right)}{W}
$$

where $W$ is the bandwidth allocated to each group. In the next section, a brief analysis on the adaptive interferernce mitigation algorithm with user grouping is presented.

\subsection{Adaptive Interference Mitigation Algorithm with User Grouping}

SIC implementation is effective for successively removing interference over a number of signals, where each signal belongs to one specific user, provided that the first signal is successfully detected. When detecting the next signal, the successfully detected signal will be taken as the 'interfering signal' to be mitigated. However, when the first signal is failed to be detected, interference mitigation is not possible to be carried out for the second signal detection. The same problem happens when any signal, not necessarily the first signal, is failed to be detected, the next signal is impossible to be detected via interference mitigation method. In order to improve performance of this interference cancellation process, an adaptive approach is introduced such that when any signal other than the last signal is failed to be detected, that the algorithm chooses another signal, which is yet to be detected, to perform interference mitigation operation and detect the signal. If the failed signal is the first signal, then the algorithm will choose another signal to be detected as the first signal. This algorithm is summarized in Figure 2.

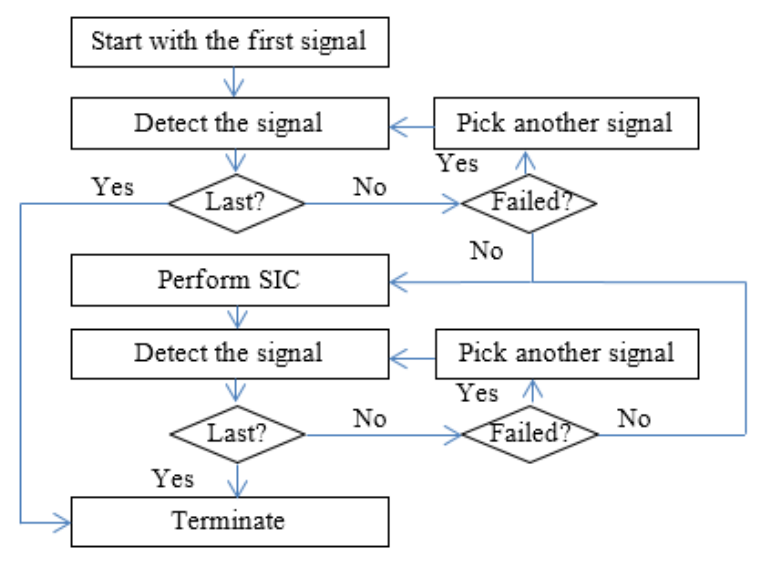

Figure 2: Adaptive Successive Interference Cancellation with User Grouping 


\section{RESULTS AND ANALYSIS}

Several tests have been carried out to observe performance of the proposed models. The measurement parameters are the energy efficiency, spectral efficiency and user energy efficiency. The physical channel propagation parameters follow the standard 3GPP LTE specifications for Pedestrian A channel model, which is specified in the same manner presented in [13-14], where P_SC $=130 \mathrm{~W}$, $\mathrm{W}=10 \mathrm{MHz},[\mathrm{N})] \_\mathrm{o}=-174 \mathrm{dBm} / \mathrm{Hz}$. The number N_u of users per group ranges between one to four and the number N_r of receiving antennas is four. The radio cells are isometric, assuming all users are at the same distance from the base station.

In Figure 3 and Figure 4, the bit error rate (BER) and the packet error rate (PER) results are plotted after running the proposed scheme, which is ImAC SIC-G to be compared with the other two existing schemes, which are Joint SIC-G [8] and AC SIC-G [13]. Each of these three schemes is separately run by setting N_u users to transmit randomly generated symbols in blocks of 12 symbols each over the uplink direction. When the symbols are received, the BER and PER are measured at the receiving antennas by computing the number of successfully detected bits over the total number of bits transmitted within all transmitted symbols, where each symbol consists of two bits. The input SNR is set in the range between -2 $\mathrm{dB}$ to $3 \mathrm{~dB}$. It can be observed from Figure 3 and Figure 4 that the proposed ImAC SIC-G scheme performed better than the two existing methods, where the recorded BER and PER are about 1dB lower than those of the existing methods when achieving BERs between $\llbracket 10 \rrbracket^{\wedge}(-1)$ to $\llbracket 10 \rrbracket^{\wedge}(-3)$. As this BER and PER reductions are observed at low input SNRs, it can be considered as useful and significant since mobile transmission is needed at low input SNRs due to certain circumstances such as the low battery level.

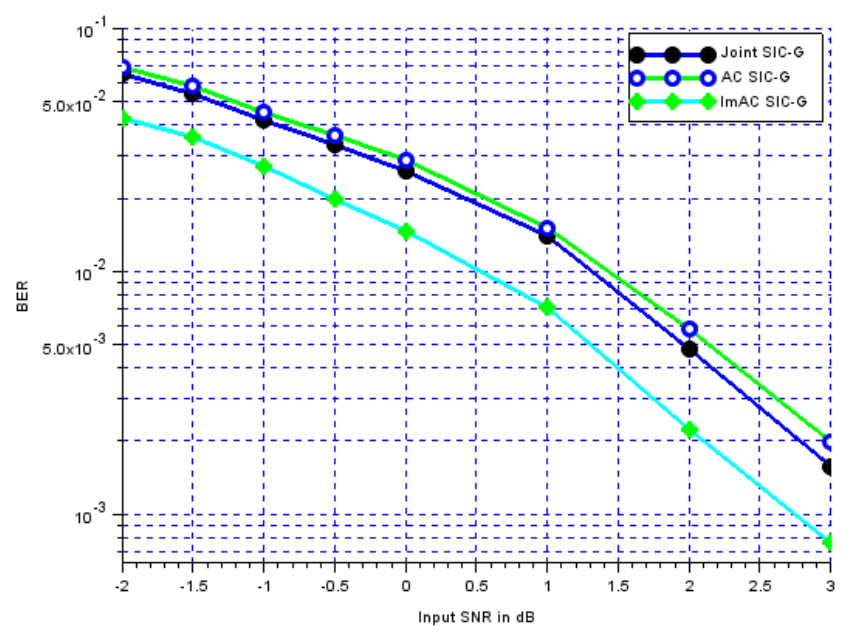

Figure 3. BER versus Input SNR

Figure. 5 shows the individual user PER observed when the test is run on the proposed ImAC SIC-G scheme and the existing Joint SIC-G. There are four users divided into two groups, each with two users. The last two digits on the name of the schemes, like Joint SIC-G12 for instance, refers to the group and the user, which in this case refers to group 1 and user 2. It is clear that the user PERs of the ImAC SIC-G scheme is lower than those of the Joint SIC-G with gains between 0.5 to $2 \mathrm{~dB}$. 


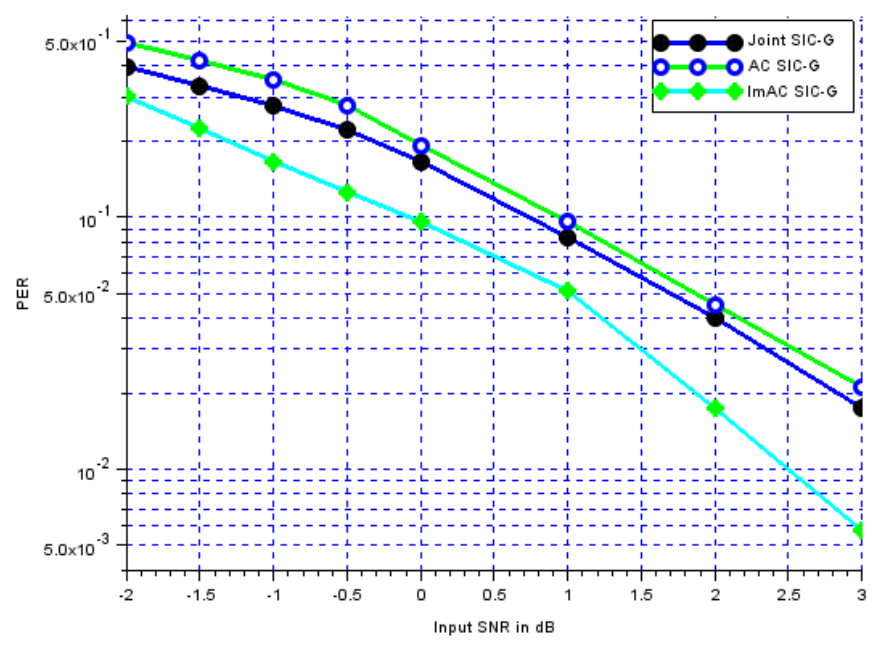

Figure 4. PER versus Input SNR.

In order to ensure that the energy efficiency increases, the interference mitigation model is proposed in this paper based on the SIC approach to increase the achievable capacity whilst reducing the incurred computational complexity. It can be seen that the proposed interference mitigation model has a gain of about $2 \mathrm{~dB}$ to $3 \mathrm{~dB}$ when a target energy efficiency of $0.3 \mathrm{Mbps} / \mathrm{Joule}$, between Figure 3 and Figure 4 . Apart from fixing the number of users per group, another test is run to observe the best grouping for a given total number of users. In this test, a total of four users are assumed in the radio cell and each group is allowed to have a different number of users.

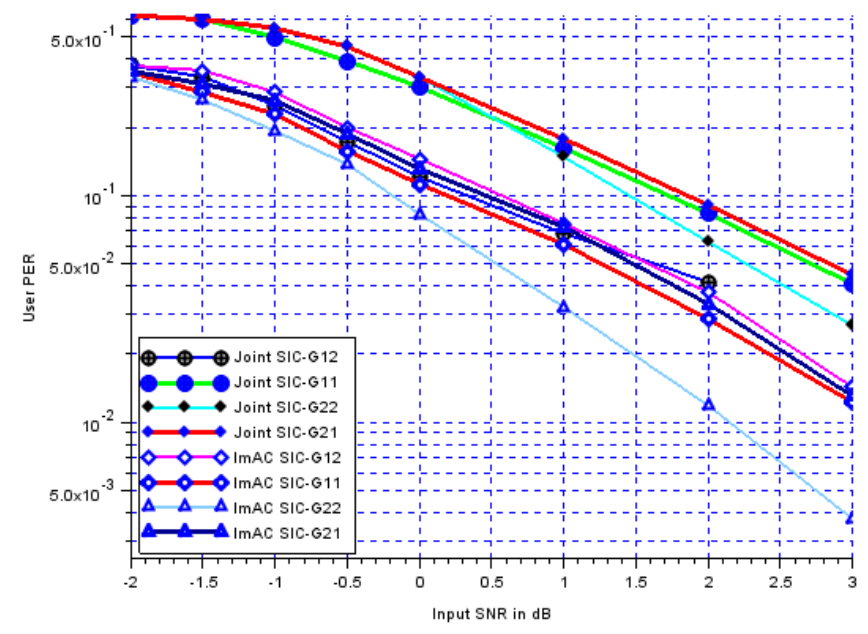

Figure 5. User PER versus input SNR.

The total energy efficiency, termed as user energy efficiency, is then calculated using Equation (10). Based on the results shown in Figure 6, it is observed that the highest user energy efficiency is obtained when each group is equally allocated with two users. The lowest user energy efficiency is when no grouping is done. The maximum number of groups in this test is two, to avoid any further increase in the spectral efficiency of the system. 


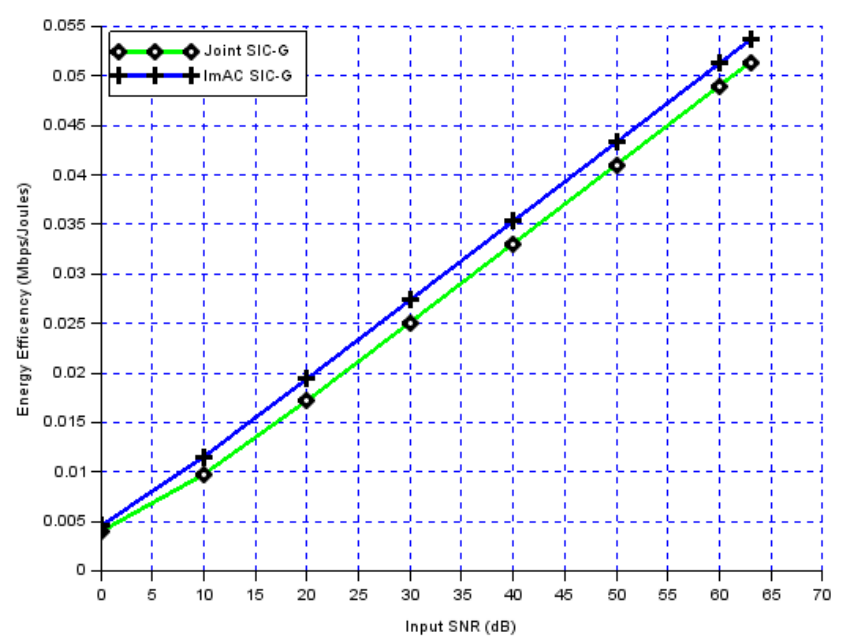

Figure 6. Energy Efficiency versus Input SNR.

Figure 6 and 7 shows the energy and spectral efficiency measured using Equation (8) and (9) respectively. The efficiency values are measured for a range of input SNRs from 0 to $60 \mathrm{~dB}$. From the two figures, it is observed that the proposed ImAC SIC-G scheme have better gains of 1 to $3 \mathrm{~dB}$. This is mainly contributed by the adaptive interference mitigation operation implemented which reduces the interference hence increasing the SINR and both the energy efficiency as well as spectral efficiency.

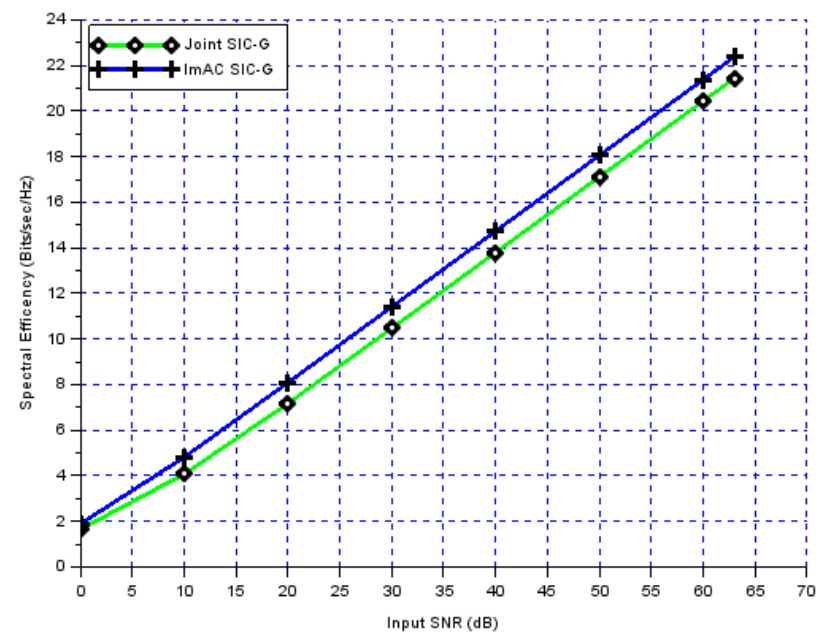

Figure 7. Spectral Efficiency versus Input SNR.

\section{CONCLUSION}

The new receiver models designed based on the proposed adaptive interference mitigation method have been demonstrated in this paper to reduce bit and packet error rates especially at low SNRs when the amount interference is considerably high. Furthermore, the energy efficiency is also improved when the interference mitigation scheme is in place, whilst increasing the spectral efficiency.

\section{ACKNOWLEDGEMENTS}

A special thanks to the Ministry of Higher Education, Malaysia, for funding this project and $\mathrm{Mr}$. Luqman Hakem for running several simulations of the proposed system presented in this paper. 


\section{REFERENCES}

[1] M. Chuan, et al, "On the performance of interference cancelation in D2D-enabled cellular networks," Wireless Communications \& Mobile Computing, vol. 16, no. 16, pp. 2619-2635, 2016.

[2] A. Idrissa, et al., "Interference and Electromagnetic Compatibility Challenges in 5G Wireless Network Deployments", Indonesian Journal of Electrical Engineering and Computer Science (IJEECS), vol. 5, no. 3, pp. 612-621, Mar 2017.

[3] I. Iskandar, et al., "Inter-cell Interference Management Technique for Multi-Cell LTE-A Network", International Journal of Electrical and Computer Engineering (IJECE), vol. 7, no. 5, pp. 2696-2705, Apr 2017.

[4] Y. Balboul and E. I. Najiba El Amrani, "Inter-cell Interference Mitigation through Flexible Resource Reuse, LPOFDM and Coordination techniques for LTE Advanced in Dense Urban Area”, Indonesian Journal of Electrical Engineering and Computer Science (IJEECS), vol. 3, no. 3, pp. 527-533, Sep 2016.

[5] R. Zakhour and D. Gesbert, "Optimized data sharing in multicell MIMO with finite backhaul capacity," IEEE Transactions on Signal Processing, vol. 59, no. 12, pp. 6102-6111, 2011.

[6] J. Hoydis, et al, "Optimal channel training in uplink network MIMO systems," IEEE Transactions on Signal Processing, vol. 59, no. 6, pp. 2824-2833, 2011.

[7] C. Park, Y.-P. Wang, G. Jongren, D. Hammarwall, "Evolution of uplink MIMO for LTE-advanced," IEEE Communications Magazine, vol. 49, no. 2, pp. 112-121, 2011.

[8] M. A. Ruder, et al, "Joint user grouping and frequency allocation for multiuser SC-FDMA transmission," Elsevier Physical Communication, vol. 8, pp. 91-103, 2013.

[9] Y. Zhou, et al, "Spectral - and energy- efficient two-stage cooperative multicast for LTE-advanced and beyond," IEEE Transactions on Wireless Communications, vol. 21, no. 2pp. 34-41, 2014.

[10] L. Xuedong, et al, "Cooperative communications with relay selection for wireless networks: design issues and applications," Wireless Communications \& Mobile Computing, vol. 13, no. 8, pp. 745-759, 2013.

[11] Y. Rui, et al, "Mode selection and power optimization for energy efficiency in uplink virtual mimo systems," IEEE Journal on Selected Areas in Communications, vol. 31, no. 5, pp. 926-936, 2013.

[12] S. R Searle, Matrix Algebra Useful for Statistics, John Wiley and Sons, 1982.

[13] J. Jiang, et al, "Energy-efficiency analysis and optimization for virtual-MIMO systems," IEEE Transactions on Vehicular Technology, vol .63, no. 5, pp. 2272-2283, 2014.

[14] H. Ab Ghani, et al, " Ant Colony Algorithm with Interference Cancellation for Cooperative Transmission", IET Signal Processing, vol. 10, no. 6, pp. 603 - 610, 2016. 


\section{BIOGRAPHIES OF AUTHORS (10 PT)}

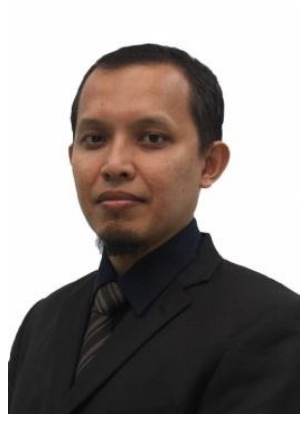

Hadhrami Ab Ghani received his bachelor degree in electronics engineering from Multimedia University Malaysia (MMU), Melaka in 2002. In 2004, he completed his masters degree in Telecommunication Engineering at The University of Melbourne. He then pursued his Ph.D. at Imperial College London in the same study area and completed his Ph.D. research in 2011. His research area of interests include wireless communication, signal processing, machine vision and artificial intelligence. Currently he serves as a senior lecturer at MMU.

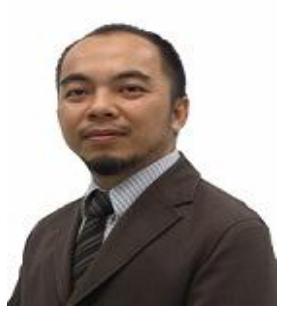

Azlan Abd Aziz received the B.S. degree in electrical and computer engineering from Ohio State University, Columbus, Ohio, USA in 1998, the M.S. degree in communication engineering from the University of Manchester, UK in 2004 and the Ph.D. degree in communication engineering from Nagoya Institute of Technology, Japan in 2012. He has many years of telecommunication industrial experience in various fields and divisions. He is currently attached to Multimedia University, Melaka in the Faculty of Engineering and Technology. His research interests are in the areas of communication theory, information theory, and networking. His current research focuses on security in wireless and vehicular ad-hoc networks.

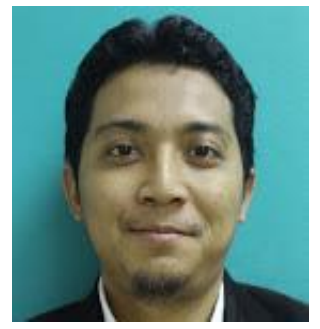

Azizul Azizan obtained his PhD qualification (2009), from University of Surrey researching on 3.5G physical layer adaptation for satellite systems. He later joined the Malaysian Communication and Multimedia Commission for more than 6 years overseeing spectrum and numbering policies and resource management. He is currently with Advanced Informatics School (AIS), Universiti Teknologi Malaysia Kuala Lumpur where his research areas includes Telecommunications, Cyberphysical Systems (inc IoT), Business Intelligence and Engineering Education. 